BIOKEMISTRI 18(2):111-119 (December 2006)

Available online at http://www.bioline.org.br/bk and at http://www.ajol.info/journals/biokem

Printed in Nigeria
An international journal published by the<smiles></smiles>

OVigerian \&ociety for Eิxperimental Siology

\title{
Dietary fatty acids alter mitochondrial phospholipid fatty acyl composition and proton leak in Drosophila melanogaster
}

\author{
Augustine OCLOO* \\ Medical Research Council Dunn Human Nutrition Unit, Hills Road, Cambridge CB2 2XY, \\ United Kingdom
}

Received 4 August 2006

MS/No BKM/2006/026, (c) 2006 Nigerian Society for Experimental Biology. All rights reserved.

\begin{abstract}
Two groups of fruit flies (Drosophila melanogaster) were maintained on different diets. Mitochondria were isolated, proton leak was measured and phospholipid fatty acid composition determined. Mitochondria from flies fed on corn-base meal (containing high amounts of polyunsaturated fatty acids, 18:2(n-6) and 18:3(n-3) and low amounts of monounsaturated fatty acid, 18:1(n-9)) contained more polyunsaturated fatty acids in their membranes than mitochondria from flies fed on yeast-base meal (containing less amount of polyunsaturates and high amount of monounsaturates). The more polyunsaturated mitochondria were leakier to protons than the more monounsaturated mitochondria.

Keywords: Fruit fly, Drosophila melanogaster, phospholipid, fatty acyl group, mitochondria, proton leak.

* Present and corresponding address: Department of Biochemistry, University of Ghana, P. O. Box LG54, Legon, Accra, Ghana, West-Africa. E-mail: augustineocloo@yahoo.co.uk, Tel: (+233) 21514086

Abbreviations used: ACL, average chain length; FCCP, p-trifluoromethoxyphenylhydrazone; MUFA, monounsaturated fatty acids; PUFA, polyunsaturated fatty acids; RCR, respiratory control ratio; SFA, saturated fatty acids; TPMP, triphenylmethyl phosphonium; UI, unsaturation index; UFA, unsaturated fatty acids.
\end{abstract}




\section{INTRODUCTION}

The 'fluid-mosaic' model of biological membranes, which visualised integral membrane proteins as 'icebergs' floating in twodimensional lipid 'sea', was proposed by Singer and Nicholson in $1972^{1}$. Although the model has since been modified and extended, it still forms the basis of modern thinking concerning cellular membranes. A simple analogy from this model is that, the phospholipid bilayer, just like the cytoplasm which forms the fluid portion of the cell in which organelles exist, provides the fluid environment in which the membrane proteins are located and forms the framework for all other membrane components. The fatty acyl groups of the membrane phospholipids constitute the core of the bilayer. They contribute to the total weight, the ultra-structure of the membrane, and play an important role in membrane function. The fatty acyl or fatty acid profile of a biological membrane refers to the type and relative amount of fatty acids that make up the membrane.

Naturally, the phospholipid fatty acyl profiles of biological membranes vary dramatically across species $^{2,3}$. For instance, the phospholpid fatty acid profiles of cellular membranes in yeasts are different from those in flies and those of mouse are different from those of rat. Interestingly, amongst these numerous variations are several consistent correlations. These correlations, have been reported over the years and have been of an immerse interest to both biochemists and physiologists. For instance, the activity of reconstituted $\mathrm{Na}^{+} / \mathrm{K}^{+}$transporter was higher in rat phospholipid liposome than in that of lizard ${ }^{4}$; the more polyunsaturated the cellular membrane of an organism the shorter its life-span ${ }^{5}$ and the smaller a mammal or a bird ${ }^{6,7}$, or the higher the thyroid hormone ${ }^{8}$, the more polyunsaturated the membrane.

The leak of protons passed the mitochondrial ATP synthetase (proton leak or uncoupling of mitochondria) ${ }^{9}$, also correlates consistently with mitochondrial phospholipid fatty acid composition. In general, the more polyunsaturated the mitochondrial membrane, the leakier it is to protons ${ }^{10}$. In addition to these consistent correlations, membrane fatty acyl composition affects many important membrane properties. Changes in membrane fatty acids are known to affect membrane fluidity ${ }^{11-13}$, permeability, and membrane liquid-gel transition temperatures ${ }^{14}$.

Functionally, membrane fatty acyl groups have significant effect on both the activity of membrane proteins and the function of the membrane $e^{15,16}$. In isolated mitochondria of $S$. cerevisiae the activities of cytochrome oxidase, NADH oxidase, succinate oxidase and ATPase vary with the degree of unsaturation, with the oxidative activity completely lost at 5\% unsaturation $^{17,18}$. In mammals, the ATPase ${ }^{19}$, the phosphate transporter ${ }^{20}$, and cytochrome oxidase are sensitive to the ratio of n-6/n-3 fatty acids ${ }^{21}$.

The consistent correlation between proton leak and mitochondrial phospholipid fatty acid composition, raises the possibility that either changes in proton leak are caused by changes in fatty acid composition or vice versa. As reported previously, changes in proton leak were not always associated with significant alteration in mitochondrial phospholipid fatty acyl composition ${ }^{9}$. Previous studies have also shown that whilst the leak through intact mitochondria correlated with membrane phospholipid fatty acyl composition, leak through liposomes accounted for just about 25\% of leak through intact mitochondria and did not correlate with mitochondria phospholipid fatty acid composition $^{3}$, indicating the importance of other membrane components and properties.

More so, although membrane fatty acyl composition has significant effects on membrane structure and function, it appears the effects of membrane fatty acyl composition on function do not follow specific pattern ${ }^{16}$. For instance, in mitochondria, there are inconsistencies in the effect of mitochondrial phospholipid fatty acyl composition on mitochondrial respiration. While some groups reported significant changes in respiration, such as increase in membrane 
potential and decrease in respiratory control ratio with high membrane unsaturation ${ }^{22,23}$, others did not report any significant changes or reported the opposite ${ }^{24-26}$. This may partly be due to the fact that most of these studies did not measure the correct mitochondrial membrane parameters, for instance the mitochondrial membrane proton conductance, which shows significant consistent correlation with mitochondrial membrane fatty acyl composition.

The aim of the present study is to find out whether the significant changes in mitochondrial membrane phospholipid fatty acid composition in response to dietary fatty acids that have been reported in other organisms, occur in the fruit fly and whether these changes have any effect on mitochondrial proton leak.

\section{EXPERIMENTAL}

Two groups of wild type (Dahomey) flies (Drosophila melanogaster) were maintained for the whole of their life-time on two different types of food: corn-based meal $(0.1 \% \mathrm{w} / \mathrm{v}$ corn meal, $0.09 \% \mathrm{w} / \mathrm{v}$ glucose, $0.02 \% \mathrm{w} / \mathrm{v}$ dried yeast, $0.01 \% \mathrm{w} / \mathrm{v}$ agar and $0.03 \% \mathrm{v} / \mathrm{v}$ nipagin in absolute ethanol (stock $100 \mathrm{~g} / \mathrm{l}$ ) per litre) and yeast-based meal $(0.1 \% \mathrm{w} / \mathrm{v}$ dried yeast , $0.1 \%$ w/v sugar (dextrose), $0.02 \%$ w/v agar, $0.03 \% \mathrm{v} / \mathrm{v}$ nipagin in absolute ethanol (stock $100 \mathrm{~g} / \mathrm{l}$ ) and $0.003 \% \mathrm{v} / \mathrm{v}$ propionic acid per litre). The flies were housed at $25^{\circ} \mathrm{C}$ under a 12-hour light: 12hour dark cycle in a Sanyo versatile environmental test chamber maintained at a constant humidity. They were maintained at a density of approximately 200 flies per $250 \mathrm{ml}$ plastic bottle (plugged with polyurethane foam bungs) and were transferred to fresh food after every two days.

\section{Sorting of flies}

Flies were sorted under $\mathrm{CO}_{2}$ anaesthesia, on a platform through which a stream of humified $\mathrm{CO}_{2}$ was passed. Healthy adult flies (mainly female) were used for proton leak experiments. This was necessary in order to obtain wellcoupled mitochondria. They were sorted at least $24 \mathrm{~h}$ before mitochondria preparation and proton leak measurement done within at most $2 \mathrm{~h}$ after mitochondria isolation.

\section{Isolation of mitochondria from Drosophila}

Fly mitochondria were isolated using standard methods as described previously ${ }^{27}$. Isolation was by two-step crushing: about 300-400 flies were inactivated, by keeping them on ice for a short period. They were then weighed in a preweighed container (the total number of flies is estimated based on $0.1 \mathrm{~g}$ per 100 flies) and then transferred into a mortar kept on ice. About 100$150 \mu \mathrm{l}$ of ice-cold isolating buffer $(250 \mathrm{mM}$ Sucrose, $5 \mathrm{mM}$ Tris-base, $2 \mathrm{mM}$ EGTA and 1\% w/v BSA, $\mathrm{pH} 7.4$ at $4^{\circ} \mathrm{C}$ ) was added. The flies were pressed down gently with a pestle to break the soft abdomen to release digestive enzymes. A colourless liquid is produced and this was discarded. A small amount (200-300 $\mu \mathrm{l})$ of isolating buffer was then added and the flies were crushed harder. This results in coloured liquid (reddish or brownish depending on the mutant). More isolating buffer (about $2 \mathrm{ml}$ ) was added and the suspension filtered through two layers of muslin into a centrifuge tube. More isolating buffer was added to two-thirds the depth of the tube and centrifuged at $4^{\circ} \mathrm{C}$ for 3 minutes (speed $=152 \mathrm{~g}$ ). The supernatant was transferred through one layer of muslin into fresh centrifuge tube and centrifuged at $8670 \mathrm{~g}$ for 10 minutes. The supernatant was discarded and mitochondrial pellet was re-suspended by adding $10 \mu \mathrm{l}$ of buffer per 100 flies.

\section{Determination of protein concentration}

Protein concentration was determined using a Bio-Rad Dc protein assay kit (Bio-Rad, Richmond, CA, USA), with bovine serum albumin as standard. Mitochondria were diluted 1 in 50 with milli-Q water. Bio-Rad's reagent $S$ and reagent $B$ were mixed in a ratio of $1: 49 \mathrm{v} / \mathrm{v}$ and the mixture called reagent $\mathrm{A} 1$. To $5 \mu \mathrm{l}$ of the diluted sample was added $25 \mu \mathrm{l}$ of A1. $200 \mu \mathrm{l}$ of reagent $\mathrm{B}$ was added and the mixture left to stand for $10 \mathrm{~min}$. Bovine serum albumin protein standards $(0-1.2 \mathrm{mg} / \mathrm{ml})$ were treated the same, and absorbance read at $710 \mathrm{~nm}$ against BSA blank to correct for proteins due to isolation medium. 


\section{Mitochondrial proton leak measurement in fruit flies (Drosophila melanogaster)}

Respiration rate and membrane potential were determined simultaneously using electrodes sensitive to oxygen and to the potentialdependent probe, triphenylmethyl phosphonium cation $\left(\mathrm{TPMP}^{+}\right)^{28}$.

Respiration rate was measured using a $2.5 \mathrm{ml}$ Clark-type oxygen electrode (Hansatech, King's Lynn, Norfolk, U.K.) maintained at $25{ }^{\circ} \mathrm{C}$ and connected to chart recorder. It was calibrated with air-saturated assay medium $(120 \mathrm{mM} \mathrm{KCl}$, $5 \mathrm{mM} \mathrm{KH} \mathrm{PO}_{4}, 3 \mathrm{mM}$ Hepes, $1 \mathrm{mM}$ EGTA, 1 $\mathrm{mM} \mathrm{MgCl} 2,0.3 \%$ w/v BSA, pH 7.2), which was assumed to contain $479 \mathrm{nmol} \mathrm{O} / \mathrm{ml}$ at $25{ }^{\circ} \mathrm{C}^{28}$. Electrode linearity from $100-0 \%$ air saturation was checked routinely by following the uncoupled respiration rate in the presence of $\quad 0.4 \quad \mu \mathrm{M} \quad$ carbonylcyanide p-trifluoromethoxyphenylhydrazone (FCCP).

Briefly, fly mitochondria $(0.3 \mathrm{mg}$ of protein $/ \mathrm{ml})$ were incubated in $2.5 \mathrm{ml}$ of the air-saturated assay medium. $5 \mu \mathrm{M}$ rotenone (to inhibit complex I), $43 \mathrm{ng} / \mathrm{ml}$ nigericin (to collapse the difference in $\mathrm{pH}$ across the inner membrane) and $1 \mu \mathrm{g} / \mathrm{ml}$ oligomycin (to inhibit ATP synthesis, so that all oxygen consumption is attributable to proton leak and not to ATP synthesis) were added to the medium before addition of mitochondria. The TPMP $^{+}$electrode was then calibrated with sequential additions of $1 \mu \mathrm{M}$ $\mathrm{TPMP}^{+}$up to $4 \mu \mathrm{M} \mathrm{TPMP}^{+}$, and $20 \mathrm{mM}$ snglycerol-3-phosphate (sodium salt) was added to start the reaction.

Respiration and potential were inhibited progressively through successive steady states by additions of potassium cyanide up to 100 $\mu \mathrm{M}$. At the end of each run $0.4 \mu \mathrm{M}$ FCCP was added to dissipate the membrane potential and release all $\mathrm{TPMP}^{+}$.

A binding correction of 0.24 was used for membrane potential calculation. Membrane potential was calculated as, $\Delta \Psi=59.1 \mathrm{log}$ (matrix $\left.\left[\mathrm{TPMP}^{+}\right]\right) \mathrm{X} \mathrm{TPMP}^{+}$binding correction/ external $\left[\mathrm{TPMP}^{+}\right]\left(\text {at } 25^{\circ} \mathrm{C}\right)^{28}$.

\section{Phospholipid extraction and fatty acid analysis}

Mitochondrial lipid extraction, phospholipids isolation and fatty acid group analysis were carried out as described previously ${ }^{9}$.

Total mitochondrial lipids were extracted in 10 $\mathrm{ml}$ of $2: 1 \mathrm{v} / \mathrm{v}$ chloroform-methanol containing $0.05 \% \mathrm{w} / \mathrm{v}$ butylated hydroxytoluene. Phospholipids were isolated by silicic acid chromatography.

Fatty acid methyl esters were prepared in the presence of boron trifluoride in methanol. They were purified on a florisil column, dried under nitrogen, re-dissolved in n-hexane and analysed on a gas chromatograph. Fatty acid methyl esters were detected using a flame ionisation detector and identified against fatty acid methyl ester standards. Amounts were measured by integration of peak areas after baseline correction and mol\% of each fatty acid was calculated as (100 x mole fa)/(sum of mole fa for all fa). No unidentified peak contributed more than $0.1 \%$ of the total fatty acid methyl esters present. Unsaturation index (UI) was calculated as $[(\Sigma \mathrm{mol} \%$ monoenoic $)+(2 \times \Sigma \mathrm{mol} \%$ dienoic $)$ $+(3 \mathrm{x} \Sigma \mathrm{mol} \%$ trienoic $)+(4 \mathrm{x} \Sigma \mathrm{mol} \%$ tetraenoic $)+(5 \times \Sigma m o l \%$ pentaenoic $)+(6 \mathrm{x}$ $\Sigma$ mol\% hexaenoic)].

\section{Statistical calculation}

Student t-test was used for statistical calculation and limit of significant set at 0.05 .

\section{RESULTS}

\section{Effect of dietary fatty acid composition on mitochondrial phospholipid fatty acyl profile}

The fatty acid compositions of total lipids from corn-based meal and yeast-base meal are as shown in Table 1. There were more polyunsaturated fatty acids $(18: 2(n-6)$ and $18: 3(n-3))$ in corn-based meal than in yeastbased meal. The yeast-based meal was higher in monounsaturated fatty acids than corn-based meal. The high 18:2(n-6) content of corn-based meal is typical of plant lipids and has been reported previously ${ }^{30-32}$. 
The fatty acyl groups in the yeast-based diet are typical of yeast cells, as was earlier reported ${ }^{2,9}$.

Table 1: Total dietary lipid fatty acyl group composition

\begin{tabular}{lcc} 
Fatty acid & $\begin{array}{c}\text { Corn-based } \\
\text { meal (Mol\%) }\end{array}$ & $\begin{array}{c}\text { Yeast-based } \\
\text { meal (Mol\%) }\end{array}$ \\
\hline $14: 0$ & 0.11 & 0.47 \\
$16: 0$ & 14.85 & 12.52 \\
$16: 1(n-7)$ & 2.11 & 44.14 \\
$16: 3(n-4)$ & $\mathrm{ND}$ & 1.386 \\
$18: 0$ & 2.68 & 3.56 \\
$18: 1(n-9)$ & 26.07 & 35.34 \\
$18: 1(n-7)$ & 0.80 & 1.15 \\
$18: 2(n-6)$ & 51.63 & 1.43 \\
$18: 3(n-3)$ & 1.75 & 0.47 \\
\hline $\begin{array}{l}\text { Values are } \\
\text { experiments. ND means of two }\end{array}$ & independent
\end{tabular}

Table 2: Global phopsholipid fatty acyl group parameters of mitochondria from Drosophila

\begin{tabular}{lll}
\hline Index & $\begin{array}{l}\text { Flies fed on } \\
\text { corn-based } \\
\text { meal }\end{array}$ & $\begin{array}{l}\text { Flies fed on } \\
\text { yeast-based } \\
\text { meal }\end{array}$ \\
\hline SFA (Mol\%) & $13.5 \pm 0.6$ & $12.2 \pm 1.0$ \\
UFA (Mol\%) & $85.6 \pm 0.6$ & $87.8 \pm 1.0^{*}$ \\
MUFA (Mol\%) & $46.9 \pm 1.5$ & $58.5 \pm 1.2^{*}$ \\
ACL & $17.2 \pm 0.1$ & $17.3 \pm 0.3$ \\
UI & $147.0 \pm 1.4$ & $141.9 \pm 1.9^{*}$ \\
Total $n-3(\mathrm{Mol} \%)$ & $3.3 \pm 0.3$ & $1.0 \pm 0.3^{*}$ \\
Total $n$-6 (Mol\%) & $35.4 \pm 1.6$ & $28.3 \pm 1.3^{*}$ \\
PUFA (Mol\%) & $38.7 \pm 1.7$ & $29.3 \pm 1.4^{*}$ \\
\hline
\end{tabular}

Values are means \pm SEM of 9 and 11 independent experiments for flies fed on corn-bas meal and flies fed on yeast-base meal respectively. *Significant compared to the other.
The total mitochondrial phospholipid fatty acyl composition in the present studies is very similar to those of other fly groups ${ }^{33}$. The fatty acyl profiles of mitochondria from the two Drosophila groups were significantly different (Figure 1). Mitochondria from Drosophila fed on corn-based meal contain significantly higher polyunsaturates (high 18:2(n-6) and 18:3(n-3)) and lower monounsaturates (18:1(n-9) and 16:1(n-7)) than mitochondria from Drosophila fed on yeast-based meal. In mitochondria from Drosophila fed on corn-based meal, the 18:2(n6) and 18:3(n-3) levels were $124 \%$ and $338 \%$ respectively of the levels in mitochondria from Drosophila fed on yeast-based meal. 16:1(n-7) and 18:1(n-9) levels were $81 \%$ and $79 \%$ respectively in mitochondria from fruit flies fed on corn-based meal, compared to flies fed on yeast-based meal. These fatty acyl group changes resulted in significant changes in some of the global phospholipid fatty acyl group parameters (Table 2). Total MUFA decreased and total PUFA, UI, $n-3$ and $n-6$ fatty acids increased in mitochondria from Drosophila fed on corn-based meal, compared to those from flies fed on yeast-based meal.

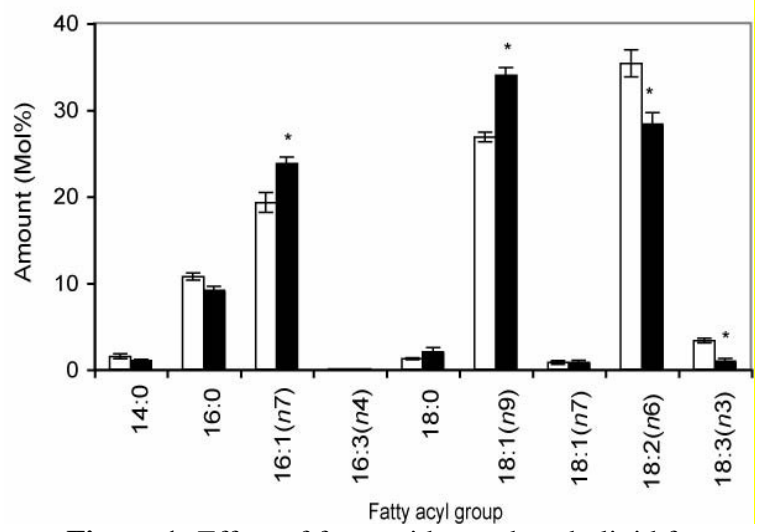

Figure 1: Effect of fatty acids on phospholipid fatty acyl composition of mitochondria from $D$. melanogaster. Two groups of Drosophila were fed on different diets (see section 2.2.3). Mitochondria were isolated and analysed. Drosophila fed on cornbase meal (white bars) compared to Drosophila fed on yeast-base meal (black bars). Values are means \pm SEM of 12 independent experiments.

\section{Effect on proton leak kinetics}

The mitochondrial proton leak kinetics in the two Drosophila groups was similar to those observed in other systems ${ }^{3,34}$. There was a nonohmic relationship between mitochondrial state- 
4 oxygen consumption and its driving force membrane potential, as reported previously, . $^{3,34}$ The membrane potentials in these flies were comparable to those of other fly groups ${ }^{33}$.

In different comparative studies, mitochondrial proton leak shows a strong negative correlation with total mitochondrial phospholipid 18:1(n-9) and total MUFA content, and positive correlation with $\mathrm{UI}^{3,32}$. In the present studies, mitochondria from flies fed on corn-based meal had lower 18:1(n-9) levels, lower total MUFA and higher UI than those from flies fed on yeastbased meal (Figure 1 and Table 2). The phospholipid fatty acyl composition differences were associated with significant changes in mitochondrial respiration (Table 3) and proton leak kinetics (Figure 2). The mitochondria from flies fed on corn-based meal were leakier to protons than mitochondria from flies fed on yeast-based meal. Mitochondria from flies fed on yeast-based meal had significantly higher state-4 and state-3 respiration rates than mitochondria from flies fed on corn-based meal. State-3 and state-4 respiration rates in mitochondria from flies fed on yeast-based meal were $120 \%$ and $126 \%$ respectively, of those of flies fed on corn-based meal (Table 3). However, respiration in presence of FCCP and respiratory control ratio (RCR) was not significantly different (Table 3). By simple extrapolation from the proton leak plot at 150 $\mathrm{mV}$, a high enough potential common to mitochondria from the two groups, mitochondria from flies fed on yeast-based meal were respiring at the rate of $15(\mathrm{nmol} \mathrm{O} / \mathrm{min} / \mathrm{mg}$ protein), while mitochondria from flies fed on yeast-base meal were respiring at 30 (nmol $\mathrm{O} / \mathrm{min} / \mathrm{mg}$ protein $)(100 \%$ increase in respiration).

Table 3: Respiration of isolated mitochondria from Drosophila

\begin{tabular}{llll}
\hline $\begin{array}{l}\text { Respiration } \\
\text { (nmol O/min/mg protein) }\end{array}$ & $\begin{array}{l}\text { Flies fed on corn-based } \\
\text { meal }\end{array}$ & $\begin{array}{l}\text { Flies fed on yeast-based } \\
\text { meal }\end{array}$ & $p$-value \\
\hline With ADP & $210.7 \pm 14.2$ & $251.5 \pm 9.1$ & 0.04 \\
With Oligomycin & $58.0 \pm 2.2$ & $73.9 \pm 1.7$ & 0.01 \\
With FCCP & $189.3 \pm 11.9$ & $204.5 \pm 8.6$ & 0.19 \\
Respiratory control ratio & $3.52 \pm 0.2$ & $3.31 \pm 0.2$ & 0.09 \\
\hline
\end{tabular}

Values are means \pm SEM of 5 independent experiments.

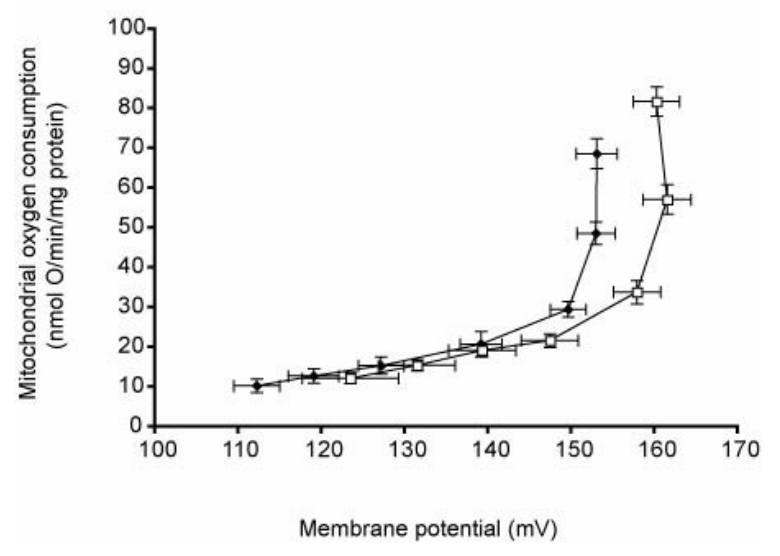

Figure 2: Mitochondrial proton leak kinetics of mitochondria from Drosophila melanogaster. D. melanogaster fed on corn-base meal (Triangles); $D$. melanogater fed on yeast-base meal (rectangles). Values are means \pm SEM of 5 independent experiments

\section{DISCUSSION}

The present studies have shown that the two fly foods have different fatty acid compositions. Dietary fatty acid manipulation is a wellestablished method known to alter membrane phospholipid fatty acyl profiles in several organisms. The effect of dietary fatty acids on cellular and sub-cellular membrane lipid fatty acid composition has been widely reported in cell cultures ${ }^{16}$, bacteria $^{35}$, yeast ${ }^{25}$, insects $^{36}$, birds $^{30}$ and mammals ${ }^{32}$. Dietary lipid modulates membrane lipid composition by two main mechanisms: (i) By providing the fatty acids available to be incorporated into membrane phospholipids, or (ii) By providing the precursors for other membrane lipid fatty acids. It has been shown clearly in the present studies that feeding Drosophila on the two different diets resulted in significant modulation in 
mitochondrial phospholipid fatty acyl composition. This is consistent with previous results $^{36}$.

In animals (especially mammals), there is a homeostatic control of membrane fatty acid composition $^{37}$, exerted at the level of gene expression $^{38}$. The result is that increasing the level of one fatty acid in the diet of an animal does not lead to high levels of just that fatty acid in the animal's membrane lipids, but it also affects the composition of other fatty acids, resulting in a fairly constant level of ratio of unsaturated to saturated fatty acids. This homeostatic control has been reported in Drosophila $^{36}$ and was apparent in the present studies.

Apart from differences in fatty acid composition, the two diets also differ in propionic acid content (see experimental). Propionic acid can provide an extra carbon source ${ }^{39}$. In isolated rat heart mitochondria, propionic acid is metabolised to tricarboxylic acid intermediates $^{39}$. This process is known to increase in presence of ATP and oligomycin ${ }^{39}$ and therefore explains the significant increase in respiration with oligomycin (state-4) or ADP (state-3) in the present studies (Table 3). However, while state- 4 respiration increased by about $20 \%$, the proton leak kinetics increased two-folds. That is even if the presence of propionic acid had any effect on mitochondrial respiration, it could only be responsible for 20$30 \%$ increase in proton leak rate at $150 \mathrm{mV}$. The remaining $60-70 \%$ increase in proton leak rate at $150 \mathrm{mV}$ must be due to some intrinsic changes in the mitochondria perhaps from the fatty acyl group differences. This statement is however debatable and could be investigated by eliminating propionic acid in the yeast meal.

The differences in proton leak observed in this study agree with the general conclusion that the more polyunsaturated the mitochondrial membrane, the higher proton conductance or the lower its membrane potential ${ }^{23}$. The differences in proton leak also correlate negatively with the level of 18:1(n-9) as seen previously ${ }^{3,34}$, suggesting strongly that these correlations are not accidental, but may have an important function relating to the proton- permeability properties of the mitochondrial membrane. While proton leak differences agree with the general observations and are consistent with general correlations, RCR and state-4 respiration differences are not. The changes in state- 4 respiration and RCR were the opposite of what is expected, thus demonstrating that using state4 and RCR to study the effect of fatty acid composition on respiration can be misleading and could be the reason for the inconsistencies in previous studies.

To conclude, the more polyunsaturated the mitochondria, the higher its proton leak.

\section{ACKNOWLEDGEMENTS}

I acknowledge the assistance of Dr Martin Brand for supervising the work. I thank the Cambridge Commonwealth and Overseas Trust, Gonville and Caius College, Cambridge and the Medical Research Council, UK for financial support.

\section{REFERENCES}

1. Singer, S. J., and Nicolson, G. L. (1972) The fluid mosaic model of the structure of cell membranes. Science 175: 720-731.

2. Daum, G. (1985) Lipids of mitochondria. Biochim. Biophys. Acta 822, 1-42.

3. Brookes, P. S., Buckingham, J. A., Tenreiro, A. M., Hulbert, A. J., and Brand, M. D. (1998) The proton permeability of the inner membrane of liver mitochondria from ectothermic and endothermic vertebrates and from obese rats: correlations with standard metabolic rate and phospholipid fatty acid composition. Comp. Biochem. Physiol. 119: B325-B334.

4. Else, P. L., and Wu, B. J. (1999) What role for membranes in determining the higher sodium pump molecular activity of mammals compared to ectotherms? J. Comp. Physiol. 169: B296-B302.

5. Pamplona, R., Portero-Otin, M., Ruiz, C., Gredilla, R., Herrero, A., and Barja, G. (2000) Double bond content of phospholipids and lipid peroxidation negatively correlate with maximum longevity in the heart of mammals. Mech. Ageing Dev. 112, 169-183. 
6. Porter, R. K., Hulbert, A. J., and Brand, M. D. (1996) Allometry of mitochondrial proton leak: influence of membrane surface area and fatty acid composition. Am. J. Physiol. 271: R1550-R1560.

7. Hulbert, A. J., Faulks, S., Buttemer, W. A., and Else, P. L. (2002) Acyl composition of muscle membranes varies with body size in birds. J. Exp. Biol. 205: 3561-3569.

8. Brand, M. D., Steverding, D., Kadenbach, B., Stevenson, P. M., and Hafner, R. P. (1992) The mechanism of the increase in mitochondrial proton permeability induced by thyroid hormones. Eur. J. Biochem. 206: 775781.

9. Ocloo, A. (2005) Uncoupling protein 2 and 3 alter mitochondrial phospholipid fatty acyl composition in yeast. Biokemistri 17: 83-94.

10. Brand, M. D., Nigel, T., Ocloo, A., Else, P. L., and Hulbert, A. J. (2003) Proton conductance and fatty acyl composition of liver mitochondria correlates with body mass in birds. Biochem. J. 376:741-748.

11. D'Antuono, C. L., and Sterin-Speziale, N. B. (1999) Changes in rat papilla microsomal membrane fluidity during development. Biol. Neonate 75, 300309.

12. Logue, J. A., Vigh, L., Joo, F., and Cossins, A. R. (1998) Catalytic hydrogenation of polyunsaturated biological membranes: effects on membrane fatty acid composition and physical properties. Biochim. Biophys. Acta 1368: 41-51.

13. Senault, C., Yazbeck, J., Goubern, M., Portet, R., Vincent, M., and Gallay, J. (1990) Relation between membrane phospholipid composition, fluidity and function in mitochondria of rat brown adipose tissue. Effect of thermal adaptation and essential fatty acid deficiency. Biochim. Biophys. Acta 1023, 283-289.

14. Vigh, L., Maresca, B., and Harwood, J. L. (1998) Does the membrane's physical state control the expression of heat shock and other genes? Trends Biochem. Sci. 23: 369-374.

15. Carruthers, A. a. M., D.L. (1986) How bilayer lipids affect membrane protein activity. Trends Bichem. 11: 331-335.

16. Spector, A. A., and Yorek, M. A. (1985) Membrane lipid composition and cellular function. J. Lipid Res. 26: 10151035.

17. Haslam, J. M., Proudlock, J. W., and Linnane, A. W. (1971) Biogenesis of mitochondria 20 the effects of altered membrane lipid composition on mitochondrial oxidative phosphorylation in Saccharomyces cerevisae. Bioenerg. 2: 351-370.

18. Watson, K., Houghton, R. L., Bertoli, E., and Griffiths, D. E. (1975) Membranelipid unsaturation and mitochondrial function in Saccharomyces cerevisiae. Biochem. J. 146: 409-416.

19. Paradies, G., and Ruggiero, F. M. (1991) Effect of aging on the activity of the phosphate carrier and on the lipid composition in rat liver mitochondria. Arch. Biochem. Biophys. 284: 332-337.

20. Paradies, G., Ruggiero, F. M., and Dinoi, P. (1991) The influence of hypothyroidism on the transport of phosphate and on the lipid composition in rat-liver mitochondria. Biochim. Biophys. Acta 1070: 180-186.

21. Stillwell, W., and Wassall, S. R. (2003) Docosahexaenoic acid: membrane properties of a unique fatty acid. Chem. Phys. Lipids 126: 1-27.

22. Pepe, S., Tsuchiya, N., Lakatta, E. G., and Hansford, R. G. (1999) PUFA and aging modulate cardiac mitochondrial membrane lipid composition and $\mathrm{Ca}^{2+}$ activation of PDH. Am. J. Physiol. 276, H149-H158.

23. Stillwell, W., Jenski, L. J., Crump, F. T., and Ehringer, W. (1997) Effect of docosahexaenoic acid on mouse mitochondrial membrane properties. Lipids 32: 497-506.

24. Fontaine, E. M., Moussa, M., Devin, A., Garcia, J., Ghisolfi, J., Rigoulet, M., and Leverve, X. M. (1996) Effect of 
polyunsaturated fatty acids deficiency on oxidative phosphorylation in rat liver mitochondria. Biochim. Biophys. Acta 1276, 181-187.

25. Proudlock, J. W., Haslam, J. M., and Linnane, A. W. (1971) Biogenesis of mitochondria. 19. The effects of unsaturated fatty acid depletion on the lipid composition and energy metabolism of a fatty acid desaturase mutant of Saccharomyces cerevisiae. $J$. Bioenerg. 2: 327-349.

26. Rafael, J., Patzelt, J., Schafer, H., and Elmadfa, I. (1984) The effect of essential fatty acid deficiency on basal respiration and function of liver mitochondria in rats. J. Nutr. 114: 255262.

27. Miwa, S., St-Pierre, J., Partridge, L., and Brand, M. D. (2003) Superoxide and hydrogen peroxide production by Drosophila mitochondria. Free Radic. Biol. Med. 35: 938-948.

28. Brand, M. D. (1995) Measurement of mitochondrial protonmotive force in Bioenergetics A practical approach. (Brown, G. C., and Cooper, C. E. eds) pp 39-62. Oxford, IRL press.

29. Reynafarje, B., Costa, L. E., and Lehninger, A. L. (1985) $\mathrm{O}_{2}$ solubility in aqueous media determined by a kinetic method. Anal Biochem. 145: 406-418.

30. Chainier, F., Roussel, D., Georges, B., Meister, R., Rouanet, J. L., Duchamp, C., and Barre, H. (2000) Cold acclimation or grapeseed oil feeding affects phospholipid composition and mitochondrial function in duckling skeletal muscle. Lipids 35: 1099-1106.

31. Rao, C. V., Zang, E., and Reddy, B. S. (1993) Effect of high fat corn oil, olive oil and fish oil on phospholipid fatty acid composition in male F344 rats. Lipids 28: 441-447.

32. Zara, V., Giudetti, A. M., Siculella, L., Palmieri, F., and Gnoni, G. V. (2001)
Covariance of tricarboxylate carrier activity and lipogenesis in liver of polyunsaturated fatty acid (n-6) fed rats. Eur. J. Biochem. 268: 5734-5739.

33. Brand, M. D., Pakay, J. L., Ocloo, A., Kokoszka, J., Wallace, D. C., Brookes, P. S., and Cornwall, E. J. (2005) The basal proton conductance of mitochondria depends on adenine nucleotide translocase content. Biochem. J. 392: 353-362.

34. Hulbert, A. J., Else, P. L., Manolis, S. C., and Brand, M. D. (2002) Proton leak in hepatocytes and liver mitochondria from archosaurs (crocodiles) and allometric relationships for ectotherms. J. Comp. Physiol. 172: B387-B397.

35. Jenkins, J. K., and Courtney, P. D. (2003) Lactobacillus growth and membrane composition in the presence of linoleic or conjugated linoleic acid. Can. J. Microbiol. 49: 51-57.

36. Stark, W. S., Lin, T. N., Brackhahn, D., Christianson, J. S., and Sun, G. Y. (1993) Fatty acids in the lipids of Drosophila heads: effects of visual mutants, carotenoid deprivation and dietary fatty acids. Lipids 28: 345-350.

37. Gibson, R. A., McMurchie, E. J., Charnock, J. S., and Kneebone, G. M. (1984) Homeostatic control of membrane fatty acid composition in the rat after dietary lipid treatment. Lipids 19: 942-951.

38. Thewke, D., Kramer, M., and Sinensky, M. S. (2000) Transcriptional homeostatic control of membrane lipid composition. Biochem. Biophys. Res. Commun. 273: 1-4.

39. Hiltunen, J. K., Kauppinen, R. A., Nuutinen, E. M., Peuhkurinen, K. J., and Hassinen, I. E. (1980) Isolated rat heart mitochondria are able to metabolize pent-4-enoate to tricarboxylic acid-cycle intermediates. Biochem. J. 188: 725-729. 